American Journal of Infectious Diseases 3 (2): 98-109, 2007

ISSN 1553-6203

(C) 2007 Science Publications

\title{
Vibrio cholerae 01 Strains of Different Ribotypes have Similar hlyA RFLP Patterns but Different Vacuolating Ability
}

\author{
${ }^{1}$ Jorge E. Vidal, ${ }^{2}$ Paula Figueroa-Arredondo, ${ }^{3}$ Silvia Giono-Cerezo, ${ }^{3}$ Rosa María Ribas-Jaimes, ${ }^{1}$ Fernando \\ Enríquez-Rincón \\ ${ }^{1}$ Departmento de Biología Celular, CINVESTAV-IPN, México DF 07360, México \\ ${ }^{2}$ Programa Institucional de Biomedicina Molecular, ENMH-IPN, México DF 07320, México and \\ ${ }^{3}$ Departmento de Microbiología, ENCB-IPN, México DF 06400, México.
}

\begin{abstract}
Extensive cytoplasmic vacuolation on Vero and HeLa cells in vitro by the Vibrio cholerae pore forming toxin HlyA, has been previously reported by our group. Vibrio cholerae $\mathrm{O} 1$ and non-O1 pathogenic strains show differences in the potential to induce vacuolation, here we study occurring variations on vacuolating cytotoxic ability, related to changes in the nucleotide sequence of the $h l y \mathrm{~A}$ orf. A collection of eight toxigenic strains of $V$. cholerae $\mathrm{O} 1 \mathrm{El}$ Tor and a non-toxigenic one, all belonging to different ribotypes was tested for their vacuolating ability, and hlyA-orf similarity based on PCR and RFLPs. The strains had extremely different vacuolating capacities, those from the ribotype 2 isolated from the US Gulf Coast, showed the highest vacuolating titer (10240 dil), and the rest of the collection had considerably lower titers ranging among 40 to 360 dilutions. PCR of hlyAorf, was performed and RFLPs were generated using seven restriction enzymes, this approach later revealed small changes of restriction maps, among the strains. The phenogram constructed from the RFLPs, showed two major branches, one of them included most of the strains, the other separates the only Mexican wild type non-O1 Vibrio cholerae. To test for vacuolating ability out of the Vibrio genetic context, the amplified hlyA-orfs from the collection of strains were cloned in pGEMT- vector system and supernatants from the recombinant $E$ coli DH5- $\square$, showed no differences on vacuolating titers, the clones always were low producers. Results from the cloning, together with those from the phenogram indicated that the hlyA gene is mainly conserved and the differences on vacuolating activity are unrelated to minute changes seen in the hlyA-orf. Production of high vacuolating titers on Vibrio strains could be due to transcriptional regulation. Whether the high vacuolating titer would be related to increased virulence, is still to be found.
\end{abstract}

Keywords: Hemolysin/Vibrio cholerae cytolysin, vacuolation, RFLP mapping

\section{INTRODUCTION}

Cholera is a diarrheal disease prevalent in the world from ancient times and nowadays it still represents a serious potential problem in developing countries ${ }^{[1,2]}$. The etiological agent, Vibrio cholerae, produces a potent enterotoxin, the cholera toxin (CT) responsible for the lethal symptoms of this disease. However, additional toxins contributing to cholera disease have been described, for example ZOT, a toxin affecting the zonula occludens and ACE, the accessory cholera toxin, both induce ion absorption alterations and lost of electrolytes through the intestinal epithelium ${ }^{[3,4]}$. The toxic properties of $V$. cholerae hemolysin HlyA, were described previously and they include lysis of erythrocytes from several animal species, enterotoxicity and cytotoxicity with cell lethality ${ }^{[5,6]}$.

Vibrio cholerae hemolysin is encoded by the hlyA gene located in the small chromosome of $V$. cholerae El Tor ${ }^{[7,8]}$. In 2001 our group reported that supernatants from non-O1 $V$. cholerae strains induced cytoplasmic vacuole formation in Vero and HeLa cells and that the vacuolating effect was inhibited by antibodies specific to HlyA, results corroborated by other research groups [9-11]

Initially we proposed that the vacuolating effect was in fact related to the one induced by Helicobacter pylori VacA cytotoxin, but our studies found several important differences. Like VacA from Helicobacter,

Corresponding Author: $\quad$ Fernando Enríquez-Rincón, PhD, Departamento de Biología Celular, Centro de Investigación y de Estudios Avanzados del IPN, México, D.F. 07360, México Tel (55)5061-3986 Fax (55)5061-3393 
HlyA monomers also self assemble into heptamers that insert themselves into the plasma membrane forming a large anion channel ${ }^{[11]}$. Whereas vacuoles induced by VacA were originated from massive swelling of membranous compartments in the late stages of the endocytic pathway ${ }^{[11]}$. HlyA instead reaches the transGolgi network from early endosomes, then it induces the vacuolating effect and cell death ${ }^{[11,12]}$.

The so called Vibrio cholerae hemolysin has demonstrated to be a cytotoxin rather than a simple hemolysin, and it might not be a coincidence that the hly A gene is present in most of the $V$. cholerae strains although its contribution to the pathogenesis is still investigated.

Several observations were the origin of this paper. From the epidemiological point of view bizarre observations have been made, for example: during the last pandemic of $V$. cholerae O1, strains of El Tor biotype isolated in Mexico were found to be atypically non-hemolytic ${ }^{[13]}$. In another work, the complete sequence of hlyA-orf from wild type strains of $V$. cholerae O1 both hemolytic RV79 (HlyA ${ }^{+}$) and nonhemolytic $\left(\mathrm{HlyA}^{-}\right)$variants, showed to have identical hlyA genomic sequences and the level of hlyA-mRNA was rather similar, therefore the cause of the resulting hemolytic defect was reported unknown ${ }^{[14]}$.

Hemolytic and vacuolating activities are notable features of the $V$. cholerae HlyA, and some aspects about the relationship of both biological effects are currently under study.

In this work we first performed a genetic approach, to the question of whether differences on levels of the vacuolating activity observed in $V$. cholerae strains from different origin was related to changes on their hlyA open reading frame, testing by RFLP of hlyAPCR amplified fragments from a ribotyped collection available (kindly donated to INDRE by Dr. K Wachsmuth from CDC, Atlanta, Ga.). Second we tested whether the ability of causing vacuolating effect and/or hemolysis was conserved among strains from the $\mathrm{O} 1$ serogroup. Working with a collection of strains previously characterized for their diversity based on isolation from geographic origin (several countries) and because they belong to different ribotypes.

In this work we found that all the supernatants from nine pandemic strains of $V$. cholerae $\mathrm{O} 1$ growing under same conditions produced a vacuolating effect on Vero cells, at very different vacuolating titers. Consistently, strains belonging to the ribotype 2 previously reported as strongly hemolytic, had the highest vacuolating titers.
Because some supernatants had higher vacuolating activity than others, we focused ourselves in looking for possible RFLP differences of the hlyA gene, which may explain differences on vacuolating titers among the bacterial collection. Interestingly, we found that RFLP patterns of hlyA genes of $V$. cholerae strains was very conserved among the collection suggesting that hlyA sequence is actually conserved and that the probable changes occurring to the HlyA protein is low, therefore the differences in vacuolating activities can not be explained by changes induced by natural mutagenesis. The most important differences in RFLPs are shown by the non-O1 Vibrio cholerae strain used as positive control for vacuolation.

This work points out that the hemolysin gene from Vibrio cholerae $\mathrm{O} 1$ is much conserved and that this characteristic probably represents a genetic advantage for the group. Although hemolytic and vacuolating effects from this $\mathrm{O} 1$ strains were weaker, comparing to $V$. cholerae outside the $\mathrm{O} 1$ serogroup.

\section{METHODS}

Bacterial strains: Strains used in this study are listed in the Table 1. All Vibrio strains were from the Cholera Laboratory at INDRE (Instituto de Diagnóstico y Referencia Epidemiológica de México). Several strains were from CDC Atlanta Cholera Laboratory also provided by INDRE. Ribotypes of the CDC strain collection were previously reported by Popovic et al. ${ }^{[15]}$. For DNA cloning experiments Escherichia coli DH5 $\alpha$ [16] was used as the heterologous host. Transformants of bacteria were routinely grown in Luria-Bertani agar (Invitrogen) with $100 \mu \mathrm{g} / \mathrm{ml}$ ampicillin (Sigma St. Louis, Mo.).

Vacuolation and hemolytic assays: Vero cells (ATCC CCL-81) were grown in DMEM supplemented with $10 \%$ fetal bovine serum (SFB), they were trypsintreated, harvested and then seeded and grown to approximately $70-80 \%$ of confluence in flat-bottom 96-well microtiter plates (Falcon) and grown at $37^{\circ} \mathrm{C}$ in a humidified $5 \% \mathrm{CO}_{2}$ atmosphere. Cytotoxicity assays for vacuolating effects in Vero tissue cultures were done as described previously [11]. Morphologic changes and vacuolation of the cells were followed under inverted microscope (Nikon). Toxin treated cell monolayers were washed with $1 \mathrm{X}$ PBS and then fixed with absolute methanol (Baker) during $12 \mathrm{~min}$, then cells were Giemsa stained and analyzed by optic upright microscopy at different magnifications. 
Vacuolation titers were recorded following previously established criteria ${ }^{[11]}$.

Bacterial cultures were done in Craig's medium $(3 \%$ Casaminoacids, $0.4 \%$ Bacto yeast extract, $0.2 \%$ glucose and $\left.0.05 \% \mathrm{~K}_{2} \mathrm{HPO}_{4}\right)$ and the strains grown at $37^{\circ} \mathrm{C}$ for $16 \mathrm{~h}$ with (150 RP) shaking. Full-grown bacteria were pelleted and supernatants filter sterilized through $0.22 \mu \mathrm{m}$-diameter filters (Millipore, Bedford, MA). Sterile supernatants were serially diluted in DMEM (Gibco, BRL, Bethesda, Maryland) added of
$2 \%$ of SFB (Invitrogen) and used for vacuolation assays.

For hemolysis assays, Vibrio cholerae strains were seeded on blood agar (Difco laboratories, Detroit, Michigan.) containing 5\% rabbit or sheep red blood cells and incubated in conditions described by Richardson et al ${ }^{[17]}$. Hemolysis was assesed by formation of transparent halos around single colonies over a period of $48 \mathrm{~h}$. When recombinant hlyA-clones were tested for hemolysis in blood agar plates, $100 \mu \mathrm{g} / \mathrm{ml}$ ampicillin (Sigma) was added.

Table 1: Description of strains and plasmids used in this study

\begin{tabular}{|c|c|c|c|c|}
\hline \multirow[t]{2}{*}{ Strain } & \multirow[t]{2}{*}{ Description } & \multicolumn{2}{|c|}{ Cytotoxic effect } & \multirow{2}{*}{$\begin{array}{l}\text { Reference or } \\
\text { source }\end{array}$} \\
\hline & & Hemolysis $^{\mathrm{a}}$ & Vacuolation $^{\mathrm{b}}$ & \\
\hline \multicolumn{5}{|l|}{ Vibrio cholerae $\mathrm{O} 1 \mathrm{El}$ Tor } \\
\hline $2514-88$ & Ribotype 2, US Gulf Coast & + & 10240 & INDRE \\
\hline $1064-81$ & Ribotype 2, US Gulf Coast & + & 10240 & INDRE \\
\hline 2164-78 & Ribotype 2, Loussiana & + & 10240 & [15] \\
\hline C7445 & Ribotype 5, Guatemala & + & 320 & INDRE \\
\hline C6706 & Ribotype 5, Peru & + & 80 & [15] \\
\hline C7986 & Ribotype 5, Guatemala & - & 40 & INDRE \\
\hline C7754 & Ribotype 6a, Rumania & + & 40 & [15] \\
\hline $2270-77$ & Ribotype 10, Australia & + & 160 & [15] \\
\hline CM91-3 & Ribotype 12, México, $\mathrm{NT}^{\mathrm{c}}$ & + & 40 & {$[13,15]$} \\
\hline Vibrio cholerae $\mathrm{O} 1$ & Classical biotype & & & \\
\hline O395 & Reference strain & - & $>10$ & [17] \\
\hline Vibrio cholerae Non $\mathrm{O} 1$ & Wild type & & & \\
\hline 52201 & Oaxaca, México. & + & 5120 & [11] \\
\hline 69750 & Tamaulipas, Mexico & + & 160 & [11] \\
\hline 44244 & Guerrero, Mexico & + & 40 & [11] \\
\hline Vibrio parahaemolyticus WP1 & Kanagawa positive strain & + & $>10$ & [33] \\
\hline E. coli $\mathrm{DH} 5 \alpha$ & $\mathrm{K}-12$ strain & - & $>10$ & [16] \\
\hline JEV18 & E. coli $\mathrm{DH} 5 \alpha(\mathrm{pJEV} 18) \mathrm{AMP}^{\mathrm{R} \mathrm{d}}$ & + & 32 & This study \\
\hline JEV35 & E. coli $\mathrm{DH} 5 \alpha(\mathrm{pJEV} 35) \mathrm{AMP}^{\mathrm{R}}$ & + & 64 & This study \\
\hline \multicolumn{5}{|l|}{ Plasmids } \\
\hline pGEMT & $\mathrm{AMP}^{\mathrm{R}}$ & & & [34] \\
\hline pJEV18 & $\begin{array}{l}\text { pGEMT- } h l y \text { A derived from 2514-88, } \\
\qquad \text { AMP }^{\mathrm{R}}\end{array}$ & & & This study \\
\hline pJEV35 & $\begin{array}{l}\text { pGEMT- } h l y \mathrm{~A} \text { derived from CM 91- } \\
3, \mathrm{AMP}^{\mathrm{R}}\end{array}$ & & & This study \\
\hline
\end{tabular}

\footnotetext{
${ }^{a}$ Results of hemolysis on sheep and rabbit red cells (+ or -). ${ }^{b}$ Vacuolating activity is expressed as the reciprocal of the highest positive dilution causing cytoplasmic vacuolation in $50 \%$ in Vero cells. ${ }^{\mathrm{C}} \mathrm{NT}$, nontoxigenic, ${ }^{\mathrm{d}} \mathrm{AMP}^{\mathrm{R}}$, ampicillin resistance.
} 
PCR amplification of hlyA open reading frame: Genomic DNAs for templates were phenol chloroform extracted, as described by Sambrook et al ${ }^{[18]}$. Previously designed primers were used to amplify the hlyA gene ${ }^{[9,119}$ : Hly Fwd (5'-CTG TCT AGA AGT GAG GTT TAT ATG CCA AAA CTC AAT CGT) and Hly Rev (5'-CTG CTC GAG TTA GTT CAA ATC AAA TTG AAC CCC TTT CAC CAA). PCR reaction mixture was prepared as follows: $1 \mu \mathrm{l}$ of template DNA $(0.10 \mu \mathrm{g}), 0.5 \mathrm{U}$ of Taq DNA polymerase (Sigma), $1.5 \mu \mathrm{l}$ of buffer high, $2.5 \mathrm{mM}$ of each deoxynucleoside triphosphate, and 10pmol from each primer. PCR was performed in an automated (Perkin-Elmer) thermocycler for 30 cycles of $94^{\circ} \mathrm{C}$ for $1 \mathrm{~min}, 60^{\circ} \mathrm{C}$ for $1 \mathrm{~min}$, and $72^{\circ} \mathrm{C}$ for $1 \mathrm{~min}$, the final cycle included a 10 min extension step at $72^{\circ} \mathrm{C}$ to ensure full extension of the product. The presence of $2200 \mathrm{pb}$ amplified products was observed by electrophoresis on $1 \%$ agarose gel and stained with ethidium bromide.

Enzimatic digestion of $h l y \mathrm{~A}$ open reading frame fragment: After amplification, hlyA PCR products were purified by affinity columns (Qiagen). The purified DNA fragment was then digested separately, 3 $\mathrm{h}$ at $37^{\circ} \mathrm{C}$ using the buffers recommended by the manufacturer, with seven restriction enzymes: Rsal, HindIII, HincII, BamHI, EcoRI, XbaI, and XhoI (Gibco, $\mathrm{BRL}$ ). The hlyA restricted fragments yielded were electrophoresed on 3.5\% agarose gels (for RsaI and HincII digestion products) and $1 \%$ agarose (for HindIII, BamHI, EcoRI, XbaI, and XhoI). Agarose gels were examined by trans-illumination then photographed and analyzed to determine the number and size of the fragments present in each case. A 100-bp or 1-kb DNA ladder was used as molecular size standard marker.

To construct the RFLP phenogram, each DNA fragment obtained by endonuclease digestion of the hlyA gene was scored as binary data. In short, presence (1) or absence ( 0 ) of the recorded band was incorporated to a database. Coefficients of similarity were generated by the band-based method of Jaccard ${ }^{[19]}$ and a phenogram showing the similarities of the gene between strains was generated by the unweighted pair group method with arithmetic mean (UPGMA). Analysis and construction of the phenogram was made using the NTSYSpc 2.02j software. Coefficients under 0.5 are considered conserved and above 0.5 are considered diverse.

PCR-based cloning of hlyA gene from strains 251488 and CM91-3: Purified hlyA amplicons from 251488 (hyper-vacuolating) and CM91-3 strains, were ligated directionally in pGEMT-Easy vector system (Promega, Madison, Wis.) and used to transform competent cells of $E$. coli DH5 $\alpha$. Ligation was plated in blood agar added of ampicillin $(100 \mu \mathrm{g} / \mathrm{ml})$, for selection of hlyA recombinant clones. Hemolytic clones were tested for insertion of hlyA fragment by PCR and checked back for the same restriction pattern of the corresponding parental $V$. cholerae hlyA gene. PCRpositive hlyA clones were additionally confirmed by enzymatic digestion using either XbaI-XhoI (for the amplicon) or EcoRI (for the vector).

\section{RESULTS}

Vacuolating effect of the culture supernatant of $V$. cholerae 01 on Vero cells: We previously described cell vacuolation due to HlyA from non-O1 V. cholerae and other researchers supported these results observing similar effect in $V$. cholerae strains from India and Brazil ${ }^{[9,10]}$. We decided to continue our study using strains of $V$. cholerae $\mathrm{O} 1$ from the last pandemic that were isolated from different geographic origins in order to compare their $h l y \mathrm{~A}$ orf. Thus, we selected eight CTtoxigenic and one non-CT-toxigenic strain of $V$. cholerae $\mathrm{O} 1$ biotype $\mathrm{El}$ Tor (Table 1). To test for vacuolating activity, Vero cells were treated with diluted and sterile culture supernatants from each strain and the characteristic vacuolization was observed in all the strains (Fig. 1B). Supernatants from E. coli DH5 $\alpha$ (not shown) and $V$. cholerae O1 Classical biotype $\mathrm{O} 395$ (Fig. 1A) were used as negative controls and as expected, no vacuolating effect was observed. Additionally, a $V$. parahemolyticus supernatant was used for alternative cytotoxicity control and no vacuolating effect was observed (not shown) but the cytotoxic titer was recorded (Table 1). The vacuolating effect in all $V$. cholerae El Tor was evident as early as 2 $\mathrm{h}$ post incubation (Fig. 1B), vacuoles all over the cytoplasm appeared progressively after $24 \mathrm{~h}$ (not shown). Vacuolating effect showed a wide range of titers among the strains (Table 1). Isolates from US Gulf Coast (ribotype 2) had the highest titers (10,240 dil), the highest vacuolating activity correlated with the strong hemolytic ability reported before by [20]. Consistent with our previous study, we found the same hyper-vacuolating titers in non-O1 V. cholerae 52201 from Oaxaca, Mexico, used as a positive control for vacuolation ${ }^{[11]}$. Strains other than those from ribotype 2, showed low titer values, ranging among 40 to 360 dilutions (Table 1).

Notably, when using undiluted supernatans to treat the cell culture monolayers and even in the smallest dilutions incubating $2 \mathrm{~h}$ (i.e. 1:10 or 1:20), the cells became rounded and most of them detached from the microplate bottom. This effect was due likely to high 
concentrations of the cytolysin-hemolysin in the supernatant, although in the toxigenic $V$. cholerae there are toxins other than CT contributing to the observed cell death. In these experiments, the supernatants of non-CT-toxigenic $V$. cholerae O1 CM91-3 (ctx) ${ }^{[15]}$ induced cell rounding and detachment similar to the rest of the toxigenic strains.

Because some El Tor strains isolated early on the seventh pandemic were reported to lack an efficient hemolytic capacity, we tested supernatants and bacterial strains of $V$. cholerae $\mathrm{O} 1$ for hemolytic activity on $5 \%$ SRBC suspension and blood agar plates respectively (Table 1).

Most of the strains were able to cause lysis of rabbit and sheep red cells, except the ribotype 5 strain from Guatemala (C7986) which was unable to cause hemolysis in any of the systems tested (Table 1) even when the strain was growth in $3 \%$ glycerol, condition that have been reported that increases the yield of hemolysin in vitro ${ }^{[17]}$, notably this strain conserved the ability to produce low titers of cell vacuolation (40) and cell detachment.

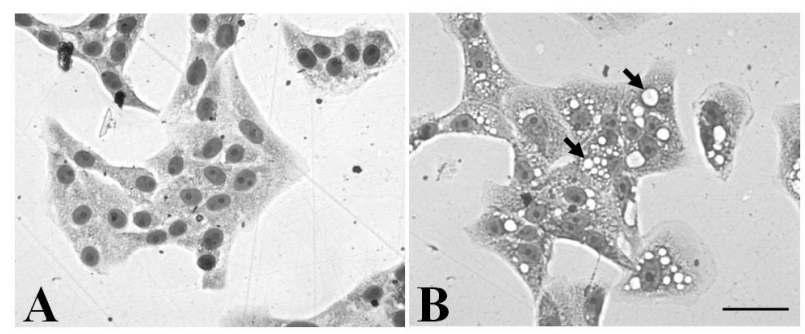

Fig. 1: Vacuolation on Vero cells induced by broth supernantant cultures of Vibrio cholerae O1 El Tor. (A) Cells treated $24 \mathrm{~h}$ with the negative control supernatant $(V$. cholerae $\mathrm{O} 1 \mathrm{O} 395$, Classical biotype) showed normal morphology, (B) vacuolated cells treated with 2514-88 El Tor strain supernatant, vacuoles were larger after $24 \mathrm{~h}$ incubation (arrows) and were formed by fusion of smaller vacuoles, eventually cells died (video microscopy data not shown). Barr in $\mathrm{B}=50 \mu \mathrm{m}$ it is valid for $\mathrm{A}$.

The $h l y \mathrm{~A}$ gene is present in vacuolating $\boldsymbol{V}$. cholerae 01 strains: The $V$. cholerae chromosomal DNA was extracted from the strains and used as template for PCR using specific primers for amplification of the hlyA open reading frame, the gene responsible for the production of the vacuolating activity.

Amplification of the expected 2,200pb fragment was observed $[14,21]$ indicating that all the toxigenic $V$. cholerae $\mathrm{O} 1$ strains harbor the vacuolating hly $\mathrm{A}$ sequence, supporting the origin of their vacuolating cytotoxin phenotype (data not shown). In agreement with our previous results, it has been described by other authors that the HlyA protein (hlyA gene product) is responsible of supernatant-induced vacuolization observed on treated Vero and HeLa cells. In these studies a $\approx 2,200 \mathrm{pb}$ hlyA gene was amplified using Indian, Brazilian and Mexican strains [9-11]. The negative controls for $V$. cholerae hemolysin PCR amplification were $E$. coli K12 DNA or $V$. parahemolyticus DNA, and they yielded not any amplicon (not shown). In addition, we used three nonO1 V. cholerae strains isolated from Mexico (Table 1), previously studied as positive control for specific hlyA$\mathrm{PCR}^{[11]}$. Once $h l y \mathrm{~A}$ amplicons from the collection were obtained, further RFLP analysis was done. Interestingly, hlyA gene of ribotype 5 strain (C7986) was amplified although the strain was not able to cause lysis of sheep or rabbit red cells; however the supernatant of the strain yielded a low vacuolating titer (Table 1).

Restriction fragment length polymorphism patterns of vacuolating $\boldsymbol{h l y} \mathrm{A}$ gene of $\boldsymbol{V}$. cholerae strains: Since we found that equally grown strains exerted different vacuolating titers, we examined whether RFLP analysis may show differences in the hlyA gene that could be interpreted as possible changes in the mature protein and hopefully correlate to the vacuolating titers observed. Purified $2200 \mathrm{pb}$ hlyA amplicons from all $V$. cholerae strains studied here were further digested separately with seven restriction enzymes, RsaI, HindIII, HincII, BamHI, EcoRI, XbaI, and XhoI, and the RFLPs observed in agarose gel electrophoresis.

Seven of the nine hlyA amplicons of $V$. cholerae $\mathrm{O} 1$ and one non-O1 $V$. cholerae (69750) strain generated a similar RFLP pattern (Fig. 2A), showing digestion fragments of expected size and suggesting that hlyA gene sequence is conserved in these strains. The size of the hlyA restriction fragments obtained were similar to those calculated directly from the published sequence and linear map of hlyA El Tor O17 and N86, (Gene Bank accession number Y00557 and X51746 respectively) (Fig. 2A) ${ }^{[21,22]}$. Strain 2270-77 (ribotype 10) generated a different $h l y \mathrm{~A}$ restriction pattern, when we used the HincII restriction enzyme an additional band of $529 \mathrm{pb}$ appeared, indicating a change in the nucleotides 392 to 397 being the HincII first recognition site predicted on hlyA, and it polymorphic site generated the next predicted fragment of $529 \mathrm{pb}$ (showed in Fig. 2C and 3A). A similar HincII restriction fragment was only observed in the RFLP from the non-O1 44244 strain (Fig. 2D). Restriction pattern of hlyA with RsaI from strain 2270-77 showed 
an approximately $557 \mathrm{pb}$ band, meaning that RsaI recognition site between 420-423 nucleotides is mutated and the next cut generated a 557 band, as was observed (Figs. 2C and 3B). The above results suggested changes in the 392 to 423 nucleotide sequence of hlyA of 2270-77 giving place to a polymorphism but it is unrelated to the activity of the vacuolating toxin.

The hlyA gene from CM91-3 strain showed an additional band of approximately $1122 \mathrm{pb}$ in the HincII pattern unlike others $V$. cholerae $\mathrm{O} 1$ strains (Figs. 2C and $3 \mathrm{~A})$. It is very probably that a polymorphic nucleotide site inside 1454 to $1459 \mathrm{pb}$ prevented the HincII restriction enzyme cut and normal C- terminal restriction pattern of 350 and 722 bands could not be noted (Fig. 3A), but a fragment of 1122 appeared instead. Interestingly, this hlyA HincII-band also could be noted in hlyA from $V$. cholerae non-O1 strains 44244 (Figs. 2D and 3A). This finding reveled that the strain 44244 shared similar polymorphic sites within hlyA gene comparing with 2270-77 and CM91-3 strains separately (Figs. 2 and $3 \mathrm{~A}$ ).



Fig. 2: Different restriction patterns of hlyA gene. Schematic representation of the restriction maps of hlyA showing in the upper solid line the digestion sites of RsaI and HincII restriction enzymes. Vacuolating genes from A) 2514-88, 1064-81, 2164-78, C7445, C6706, C7986, C7754, 69750 (non-O1) and software expected of the linear map from $\mathrm{O} 17$ and N86 V. cholerae El Tor strains, B) CM91-3, C) 2270-77, D) 44244 (non-O1). Numbers below the lines indicate base pairs of fragments expected by the software and the fragments observed actually by electrophoresis are showed in bold. Arrowhead indicates HincII polymorphic site and the asterisk points out the RsaI polymorphic site. The diamond represents a unique additional $\mathrm{X} b a \mathrm{I}$ recognition site inside the hlyA-orf of the strain 44244.

Restriction enzymes either EcoRI or BamHI did not generate any cut along hlyA gene and HindIII induced similar pattern in all strains (data not shown), as was predicted in silico. However, non-O1 strains 44244 and 52201 strains displayed difference hlyA pattern. 52210 had a site unique extra that is recognize by XhoI (not shown) yielded two fragments of near 1350 and $950 \mathrm{pb}$, whereas hlyA of 44244 had a unique additional site for $X b a I$ (Fig. 2D). Strain 69750 had an identical restriction pattern to majority of $V$. cholerae $\mathrm{O} 1$ strains (Fig. 2A). All these results indicated that hly A sequence is conserved among strains used and we suggest that high or low vacuolating capabilities of $V$. cholerae strains from different origin are probably residing outside the hlyA gene.
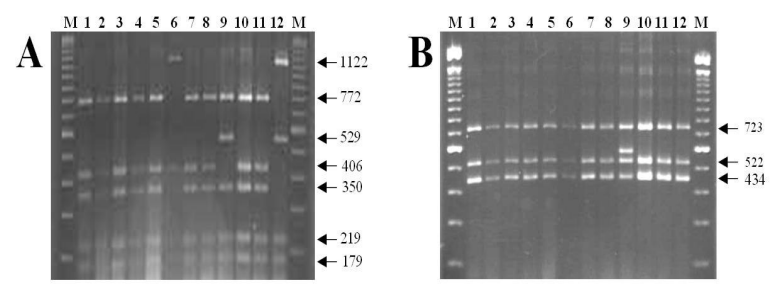

Fig. 3: RFLP-PCRs of the hlyA gene from vacuolating $V$. cholerae strains. Amplified hlyA genes were exonuclease restricted with the following enzymes: A) HincII and B) RsaI. Four $H$ HincII and two RsaI, restriction patterns were obtained from the collection of $12 \mathrm{~V}$. cholerae strains. Lane M, is $100 \mathrm{pb}$ ladder and the next lanes are the restriction analysis of the hlyA gene of the following $V$. cholerae strains, 1) 2514-88, 2) $1064-81$, 3) $\mathrm{C} 6706$, 4) 2164-78, 5) $\mathrm{C} 7754$, 6) CM91-3, 7) C7445, 8) C7986, 9) 2270-77, 10) 52201, 11) 69750, 12) 44244. Numbers at the right are base pairs and correspond to the size of the fragment obtained in each case. In B, the line 9 shows an unusual additional fragment of $557 \mathrm{pb}$.

Phenogram construction: To depict the RFLP results a data matrix was performed using each enzyme cutting along hlyA gene, these data were used to obtain the Jaccard similarity coefficients and construct a phenogram with a NTSYSpc $2 \AA$ software ${ }^{[19]}$. Phenogram showed two branches, one major branch included hlyA from both high and low vacuolating strains that displayed nearly identical RFLP patterns (Fig. 4). Inside this branch, strains 52201, 2270-77 and CM91-3 were found with slight differences among hlyA sequence, but it could not be associated to vacuolating activity.

A second branch included only one strain, $V$. cholerae non-O1 44244 (Fig. 4), this had the lowest similarity index for hlyA among the strains, however, although differences are not considerable according to Jaccard index we can group this gene in a different branch.

Recently, investigators have obtained a partial sequence of the hlyA gene from $40 \mathrm{~V}$. cholerae strains, they found an average of $3.2 \%$ difference that was 
minimum among the strains, however comparing two non-O1 strains they observed a maximum of $7.23 \%$ of differences allowing to group these strains into two distinct branches of a phylogenetic tree, in agreement with our results and phenogram obtained with our RFLP-PCR scheme ${ }^{[23]}$.

Thus, this phenogram suggest that $V$. cholerae hlyA strains used here, have a high sequence similitude and different intrinsic vacuolating capabilities that could not be correlated with RFLP pattern and phenogram.

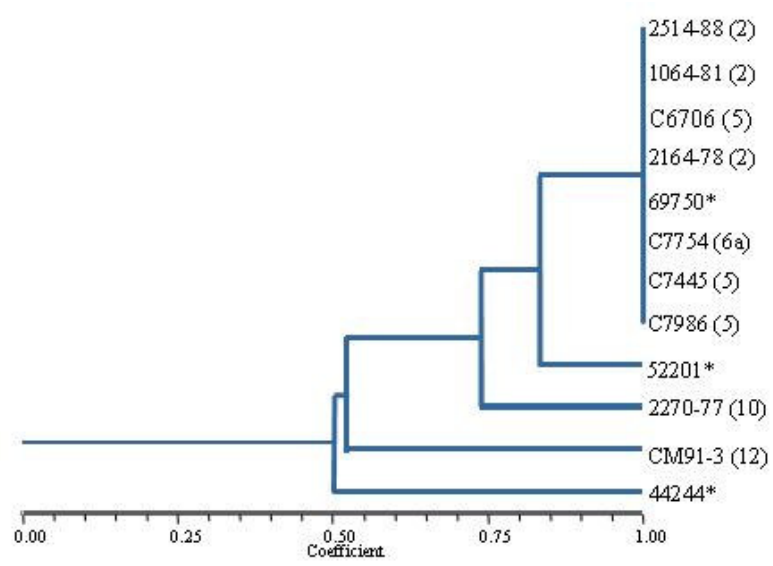

Fig. 4: Phenogram of hlyA genes from $V$. cholerae strains elaborated from the RFLP patterns experimentally obtained. Relationships were determined using Jaccard similarity coefficient. The numbers in brackets are the strain ribotype, asterisks are pointing out the Mexican non-O1 V. cholerae isolates.

hlyA cloning and expression of vacuolating phenotype from high and low vacuolating strains: To reinforce the idea that vacuolating titer is not mediated by hlyA sequence, supernatants from two hlyA recombinant strains were obtained and tested for vacuolating activity. We choose for cloning hlyA from high vacuolating strain 2514-88 (vacuolating titer 10210) of ribotype 2 and low vacuolating strain CM913 (vacuolating titer 40 ) of ribotype 12 . hlyA from these strains was amplified with primers hlyAF and hlyAR and cloning directionally into a ampicillin resistance selection vector pGEMT, then transformed $E$. coli DH5 $\alpha$ (Fig. 5A). Recombinant plasmids were called pJEV18 (cloned hlyA from 2514-88) and pJEV35 (cloned hlyA from CM91-3). Transformed strains JEV18 and JEV35 produced stable hemolytic activity on blood agar of red cell from either sheep or rabbit plus ampicillin (Fig. 5B and not shown), according with others hlyA-encoded E. coli clones reported ${ }^{[8,17,21]}$. However this hemolytic activity was much less efficient than parental $V$. cholerae (not shown), as reported elsewhere ${ }^{[24]}$. In regard to vacuolation, supernatants from recombinant clones produced similar vacuolating titers among them in spite of hlyA genes were obtained from $V$. cholerae strains with low and high vacuolating activities (Fig. 5C) suggesting that vacuolating activity depends of any other genetic element for regulation, secretion or post-transductional modification in parental strains.

\section{DISCUSSION}

The $V$. cholerae HlyA hemolytic mechanism has been largely controversial. Differences in hlyA restriction pattern were previously reported between El Tor RV79 strain and the classical strain 569B ${ }^{[14]}$, suggesting a non hemolytic defect of classical strain 569B related to the structural gene, fact that was later confirmed by sequencing, showing specifically that there was an 11 base-pair deletion in the hlyA-orf. Thereby a truncated protein $\mathrm{HlyA}^{*}$ uncapable of hemolysis is exported to the extra-cellular medium ${ }^{[14,22]}$. By working with another classical strain, we observed that the hlyA deletion in the $V$. cholerae classical strain $\mathrm{O} 395$ is enough to suppress not only the hemolytic but the vacuolating activity as well (Fig. 1A) and in fact we have chosen this strain as a negative control for our experiments.

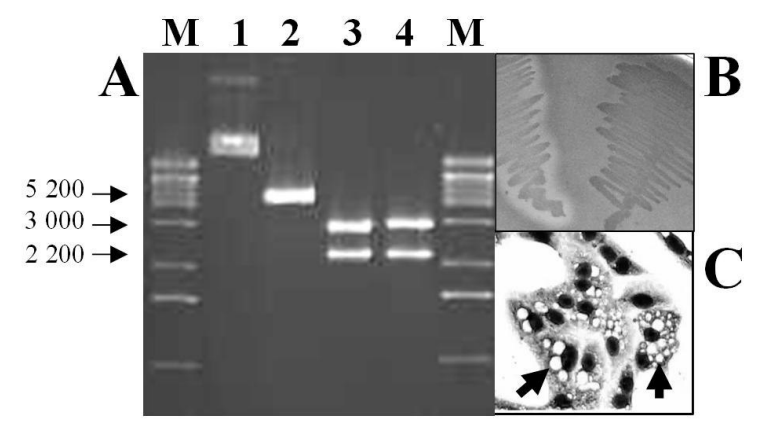

Fig. 5: Vacuolating and hemolytic activity from the recombinant plasmids containing hlyA and expressed in E. coli. A) Electrophoretic profile of pJEV18 (plasmid containing $h l y \mathrm{~A}$ from 2514-88 strain of $V$. cholerae O1), lines (M) molecular weight marker $\lambda$ HindIII-EcoRI digested, (1) plasmid extraction from recombinant JEV18 strain; (2) pJEV18 linearized with SalI. Restriction enzyme characterization (3) EcoRI (recognition site flanking the cloning multiple site of the pGEMT) and (4) XbaI-XhoI (recognition site flanking the hlyA amplicon). Numbers on the left are base pairs.B) Hemolysis on ampicillin-blood agar with 5\% sheep erythrocytes, left JEV18, right E. coli $\mathrm{DH} 5 \alpha$. with no insert (control). Bacteria were incubated aerobically at $37^{\circ} \mathrm{C}$ during 24 and $48 \mathrm{~h}$. C) Vero cells vacuolating effect exhibited by the hlyA recombinant clone JEV18, the cytotoxicity test was incubated by only 2 $\mathrm{h}$, then fixed and Giemsa stained. Arrows showed the cytoplasmic vacuolation. 
For the present study, semi-quantitative analysis of the vacuolating activity of bacterial culture supernatants in Vero cells was made, using eight toxigenic strains from the $7^{\text {th }}$ pandemic caused by El Tor biotype and a non toxigenic $V$. cholerae strain (one) and we confirmed that culture supernatants of these actually elicit vacuolating toxicity (Fig. 1B). Notably, the strains showed a wide range of vacuolating titers (Table 1). Low vacuolating titers ranged between dilutions 1:40 to 1:320. We considered as a high titer one up to 5120 or 10240 and strains yielding these titers were considered hyper-producers of vacuolating effect. Within the group of analyzed strains, three isolates from US Golf Coast of ribotype 2 yielded high vacuolating titers, while the others induced low vacuolating titer (Table 1).

Interestingly, under the assay conditions used here, hyper-vacuolating effect was obtained with highly diluted supernatants. In contrast, other investigators had to concentrate culture supernatant of their $V$. cholerae strains in order to obtain similarly high vacuolating titers (1:10240). Therefore, we used here epidemic $V$. cholerae ribotype 2 and non $\mathrm{O} 1$ V. cholerae 52201 because they showed higher vacuolating activity compared to other strains reported. So, our interest was mainly focused in finding the probable cause of this increased activity and compare the efficiency of vacuolization in epidemic and non epidemic $V$. cholerae strains ${ }^{[10]}$.

A study made by Barret and Blake ${ }^{[20]}$ reported that $V$. cholerae El Tor isolated from Louisiana in 1978 was strongly hemolytic, making them different from the rest of the world-wide El Tor isolates. Since we hypothesized that hemolytic and perhaps vacuolating activity can both be related to intrinsic characteristics that could be approached by changes of the hly A open reading frame, and that those changes could be related to final changes in the protein, we proceeded to study the hlyA-orf by performing PCR followed by RFLPs of several restriction enzymes.

In the other hand, although transcriptional regulation of HlyA by El Tor V. cholerae could be a complex event, $[8,25]$ reported data revealed that a $11.9 \mathrm{kDa}$ protein named HlyU, up-regulates expression of HlyA and probably promotes expression of some other virulence factors in vivo ${ }^{[25,26]}$.

The vacuolating effect should behave in the same way, however, the vacuolating activity could probably be also dependent on the efficiency of the HlyA protein to assemble as heptamers capable to form functional anion channels in the host cell and that the concentration of the protein reaches a critical amount of membranal pores that the cell can still repair.
Previous studies about the regulatory role of HlyU, describe how this protein stimulate similar mRNA level of hlyA in hemolytic and non-hemolytic strains, but despite the presence of the hlyA-messenger RNA, hemolysis is still missing in the non hemolytic phenotype, therefore it seems to be a defect at the level of the synthesis of the protein ${ }^{[1,14,26]}$.

Despite differences on the production of vacuolating activity, we were able to amplify the 2,200 pb hlyA gene from all the strains. An interesting observation was that the only strain being non hemolytic to rabbit or sheep red cells was able to show vacuolation (C7986 $h l y \mathrm{~A}^{+}$) (Table 1), from this fact we propose that probably the hemolytic and the vacuolating activities reside on different domains of the molecule. Unfortunately, we were unable to associate those with RFLP pattern, but instead we found the hlyA-orf to be much conserved among O1 serotype despite their otherwise distant origin indicated by ribotyping.

An alternative hypothesis fitting this result could be that an additional or associated factor to HlyA still unknown would be necessary for lysis of erythrocytes and that such factor is not produced by the non hemolytic strains, but in our hands this hypothesis is discarded after we used a specific antibody against HlyA which is sufficient to completely block both vacuolating and hemolytic activities.

Nevertheless, the fact that some $h l y \mathrm{~A}^{+}$strains can be vacuolating but not hemolytic is shown here for the first time, suggesting that hemolytic activity can be absent because it is probably a less important biologic activity than the vacuolization.

Notably, other researchers already showed that the vast majority of environmental isolates of $V$. cholerae, including epidemic toxigenic, non-toxigenic and nonO1 strains contain the hlyA gene ${ }^{[27,28]}$ suggesting that hly A might be an important gene to the microorganism survival, also in environmental conditions as much as in conditions of infection. In the other hand, environmental strains that contain an active hlyA may have a potential pathogenic advantage because the vacuolating cytotoxic phenotype could be contributing to diarrhea.

Several works mentioning the importance of HlyA in pathogenesis and diarrhea of non-toxigenic O1 and non-O1 Vibrios have been published ${ }^{[1,29]}$. In our vacuolating assays, we included a $V$. cholerae $\mathrm{O} 1$ non toxigenic El Tor strain (CM 91-3) isolated from a patient with gastrointestinal illness from Quintana Roo, Mexico. This strain belongs to the ribotype $12^{[15]}$ and lacks the $\operatorname{ct} x A B$ virulence cassette, therefore it does not secrete the ctxf virulence determinants ${ }^{[13]}$. However, 
CM91-3 strains still had the capacity of causing not cholera disease. This fact raises the possibility for the hemolytic/vacuolating associated toxin, to be probably the major toxin contributing importantly to diarrhea in these cases. Although this finding does not exclude the possibility of unknown virulence factors in these kind of strains would be causing gastrointestinal disease, but they were not then described.

We previouly studied the cytotoxicity of HlyA hemolysin from $V$. cholerae and found that this cytotoxin induces a very fast vacuolating effect in Vero cells, and that culture supernatants from several strains yield different vacuolating titers when cultured in vitro under the same conditions and at the same time. When we observed that the vacuolating activity was not proportional to the hemolytic activity, and that epidemic isolates were basically unefficiently hemolytic, meanwhile non-O1 Vibrio on the contrary, were highly hemolytic, we developed this work, to look first for differences in the open reading frames of hly $\mathrm{A}$ of dissimilar epidemic isolates and diverse geographic areas, so separated that they actually belong to different ribotypes. Here, we were able to see only no significant differences among the epidemic isolates by RFLP and few differences among the hlyA genes from non-O1 $V$. cholerae isolates. The differences that we found did not correlate with higher - or lower- production of the vacuolating titers, suggesting that no changes in the hlyA-orf are related to the biologic function. Although the nature of variation of the vacuolating capacity among strains is not clear ${ }^{[9-11]}$, this study focuses on the different vacuolating activities shown by a collection of epidemic and several non epidemic $V$. cholerae strains. Rader and Murphy ${ }^{[14]}$ reported that the nucleotide sequences of the hlyA gene from the strain RV79 $\left(\mathrm{Hly}^{+}\right)$and its variant (Hly ${ }^{-}$) were identical, they suggested that the difference altering the phenotype lies outside the structural gene ${ }^{[14]}$. Anyway, vacuolating but non hemolytic C7986 strain may be capable to produce a HlyA protein with a putative vacuolating motif that is not hemolytic. Other approaches are needed to further study this phenotypic variation.

In a recent study, Byun et al obtained the hlyA partial sequence of 33 strains of $\mathrm{O} 1 \mathrm{~V}$. cholerae, non-toxigenic $\mathrm{O} 1$ and non-O1 $V$. cholerae and they noticed polymorphic sites along hlyA gene, but minimum variation was found, concluding that the genomic sequence of hlyA is highly conserved. Our screening agrees with those data since in agreement with it, we found similar hlyA-orfs in $\mathrm{O} 1$ and non $\mathrm{O} 1$ Vibrio, based on our PCR-RFLP assay. However, they did not focus their research on HlyA vacuolating neither hemolytic activity ${ }^{[23]}$.

Despite variation in their hemolytic activity, these strains were located within the same branch in the hlyA phenogram (Fig. 4), suggesting that the hlyA genetic map, does not influence the increase of vacuolating activity observed. There is probably a regulatory element or an additional toxin associated with the increased vacuolating activity that might have a role in over-expression of the vacuolating effect and it might contribute directly to diarrhea.

Additionally, all tested strains produced lysis of the Vero cell culture when superntants were low diluted (1:20 or $1: 10)$, the same that was found in our previous work $^{[11]}$. It seems like high dosages of HlyA, induce lytic cell death, but the cell death mechanism on intoxication of the cell undergoing vacuolization still need to be elucidated.

Results of this work suggest that differences in the vacuolating ability of the $V$. cholerae hemolysin are not mediated by genetic variation at least detectable by hlyA-RFLPs. Our PCR-RFLP scheme of hlyA, points out that the vacuolating gene was nearly identical among the strains, and the groups in the phenogram were independent of the levels of vacuolating activity. There was no RFLP phenotype characterizing the high titter of vacuolating phenotype as we supposed. On the contrary, seems like the vacuolating capacity is independent from variations found by the RFLPs.

Nevertheless in this study we showed how the hlyA-orf from epidemic isolates is conserved but the non epidemic isolates tend to show more significant RFLP changes, although they are un-related to the hyperproducer phenotype.

To be able to observe efficiency of the vacuolating effect, producing the protein in a heterologous host, we additionally cloned the hlyA gene (pGEMT) and tranformed $E$. coli. The genes were, one from hypervacuolating phenotype 25214-88 (JEV18) and one from a low-vacuolating phenotype CM91-3 (JEV35). Culture supernatants from the recombinant clones yielded basically the same (mainly low) titers of the vacuolating effect in Vero cells (Fig. 5C). The genes cloned showed mostly low titers of vacuolization, disregarding their hyper-vacuolating or low vacuolating background (Table 1). Cytoplasmic vacuoles found using the cloned hlyA were physiologically similar to the ones induced by the parental strains, since two different kinds of vacuoles were HlyA-induced based on staining with neutral red dye. Despite of the different background of the parental strains, vacuolating titers of the recombinants were similar (Table 1), probably 
suggesting the need of a hypothetic transcription factor produced by $V$. cholerae that might be necessary for the high vacuolating activity. Anyway, this alternative possibility remains to be tested.

An increasing number of bacterial toxins inducing vacuolating effect have been described in the last few years. Apart from V. cholerae HlyA, others like VacA of $H$. pylori, ShlA from Serratia marscescens, aerolysin from A. hydrophila and also Sat, an auto-transporter protein from uropathogenic $E$. coli has been shown to cause vacuolating cell damage. An important observation is that almost everyone of these toxins is the major virulence factor described for its pathogen ${ }^{[11,}$ ${ }^{30-33]}$. Although the cell damage mechanism from the mentioned vacuolating toxins appears to be diverse, the presence of large cytoplasmic vacuoles at least indicates that intracellular traffic is being damaged; therefore these toxins can play a key role in mediating disease.

As a mechanism for the $V$. cholerae hemolysin, there is recent evidence supporting HlyA as a toxin that produces sub-cellular injury consisting of induction of autophagy [35], which is a defensive cell pathway that degrades cytoplasmic components, including organelles. This mechanism originally was described as a response against intracellular pathogens but it also could be a mechanism to get rid damaged organelles, such as membranous vacuoles bearing heptameric anion channels formed by the Vibrio cytolysin HlyA. Observations from our studies (video microscopy data) are also consistent with the autophagy hypothesis, because after mild treatments with the vacuolating cytotoxin, the cells undergo fusion of the large vacuoles until they eventually die, showing characteristics consistent with programmed cell death (unpublished data).

Moreover, the phenomenon of autophagy induced by HlyA, would probably be participating in gastroenteritis rather than in cholera disease, since non toxigenic but diarrheagenic $V$. cholerae strains seem to use hemolysin/cytolysin as a mechanism to induce diarrhea. Besides, since cholera toxin has been demonstrated to take advantage from the cellular traffic to transport itself towards the apical side of the enterocyte, we speculate that it would be necessary for cholera disease to keep HlyA low, so it would not interfere with the normal intracellular traffic of its target cell in order to let CT become successful.

Data shown here support that the hlyA gene is conserved since small differences evidenced by RFLPs are unrelated to the differences found in the vacuolating activity from supernatants of several strains, nevertheless, as we noticed, the production involving synthesis and/or secretion of the HlyA vacuolating cytotoxin is increased in some strains and this fact may probably be playing a role in gastroenteritis and perhaps is at least dispensable for cholera disease.

\section{REFERENCES}

1. Kaper J.B., J.G. Morris, Jr. and M.M. Levine, 1995. Cholera. Clin Microbiol Rev, 8: 48-86.

2. Lacey S.W, 1995. Cholera: calamitous past, ominous future. Clin Infect Dis, 20: 1409-1419.

3. Fasano A., B. Baudry, D.W. Pumplin, S.S. Wasserman, B.D. Tall, J.M. Ketley and J.B. Kaper, 1991. Vibrio cholerae produces a second enterotoxin, which affects intestinal tight junctions. Proc Natl Acad Sci USA, 88: 5242-5246.

4. Trucksis M., J.E. Galen, J. Michalski, A. Fasano and J.B. Kaper, 1993. Accessory cholera enterotoxin (Ace), the third toxin of a Vibrio cholerae virulence cassette. Proc Natl Acad Sci USA, 90: 5267-5271.

5. Ikigai H., A. Akatsuka, H. Tsujiyama, T. Nakae and T. Shimamura, 1996. Mechanism of membrane damage by El Tor hemolysin of Vibrio cholerae O1. Infect Immun, 64: 2968-2973.

6. Zitzer A., T.M. Wassenaar, I. Walev and S. Bhakdi, 1997. Potent membrane-permeabilizing and cytocidal action of Vibrio cholerae cytolysin on human intestinal cells. Infect Immun, 65: 12931298.

7. Heidelberg J.F., J.A. Eisen, W.C. Nelson, R.A. Clayton, M.L. Gwinn, R.J. Dodson, D.H. Haft, E.K. Hickey, J.D. Peterson, L. Umayam, S.R. Gill, K.E. Nelson, T.D. Read, H. Tettelin, D. Richardson, M.D. Ermolaeva, J. Vamathevan, S. Bass, H. Qin, I. Dragoi, P. Sellers, L. McDonald, T. Utterback, R.D. Fleishmann, W.C. Nierman and O. White, 2000. DNA sequence of both chromosomes of the cholera pathogen Vibrio cholerae. Nature, 406: 477-483.

8. Manning P.A., M.H. Brown and M.W. Heuzenroeder, 1984. Cloning of the structural gene (hlyA) for the haemolysin of Vibrio cholerae El Tor strain 017. Gene, 31: 225-231.

9. Mitra R.K., R.K. Nandy, T. Ramamurthy, S.K. Bhattacharya, S. Yamasaki, T. Shimada, Y. Takeda and G.B. Nair, 2001. Molecular characterization of rough variants of Vibrio cholerae isolated from hospitalized patients with diarrhea. J Med Microbiol, 50: 268-276. 
10. Coelho A., J.R. Andrade, A.C. Vicente and V.J. Di Rita, 2000. Cytotoxic cell vacuolating activity from Vibrio cholerae hemolysin. Infect Immun, 68: 1700-1705.

11. Figueroa-Arredondo P., J.E. Heuser, N.S. Akopyants, J.H. Morisaki, S. Giono-Cerezo, F. Enriquez-Rincon and D.E. Berg, 2001. Cell vacuolation caused by Vibrio cholerae hemolysin. Infect Immun, 69: 1613-1624.

12. Moschioni M., F. Tombola, M. de Bernard, A. Coelho, A. Zitzer, M. Zoratti and C. Montecucco, 2002. The Vibrio cholerae haemolysin anion channel is required for cell vacuolation and death. Cell Microbiol. 4: 397-409.

13. Giono-Cerezo S., M.G. Rodriguez Angeles, L. Gutierrez-Cogco and J.L. Valdespino-Gomez, 1994. Phenotypic and genotypic characterization of Vibrio cholerae O1. Rev Latinoam Microbiol, 36: 243-251.

14. Rader J.R and. A.E. Murphy, 1988. Nucleotide sequences and comparison of the hemolysin determinants of Vibrio cholerae El Tor RV79(Hly+) and RV79(Hly-) and classical 569B(Hly-). Infect Immun, 56: 1414-1419.

15. Popovic T., C. Bopp, O. Olsvik and K. Wachsmuth, 1993. Epidemiologic application of a standardized ribotype scheme for Vibrio cholerae O1. J Clin Microbiol. 31: 2474-2482.

16. Hanahan D. and M. Meselson, 1983. Plasmid screening at high colony density. Methods Enzymol, 100: 333-342.

17. Richardson K., J. Michalski and J.B. Kaper, 1986. Hemolysin production and cloning of two hemolysin determinants from classical Vibrio cholerae. Infect Immun, 54: 415-420.

18. Sambrook J., E.F. Fritsch and T. Maniatis, 1989. Molecular cloning: a laboratory manual. Cold Spring Harbor Laboratory. Cold Spring Harbor. N.Y.

19. Boldo X.M., L. Villa-Tanaca, G. Zuniga and C. Hernandez-Rodriguez, 2003. Genetic diversity among clinical isolates of Candida glabrata analyzed by randomly amplified polymorphic DNA and multilocus enzyme electrophoresis analyses. J Clin Microbiol, 41: 4799-4804.

20. Barrett T.J.and P.A. Blake, 1981. Epidemiological usefulness of changes in hemolytic activity of Vibrio cholerae biotype El Tor during the seventh pandemic. J Clin Microbiol, 13: 126-129.
21. Yamamoto K., Y. Ichinose, H. Shinagawa, K. Makino, A. Nakata, M. Iwanaga, T. Honda and T. Miwatani, 1990. Two-step processing for activation of the cytolysin/hemolysin of Vibrio cholerae $\mathrm{O} 1$ biotype El Tor: nucleotide sequence of the structural gene (hlyA) and characterization of the processed products. Infect Immun, 58: 4106-4116.

22. Alm R.A., U.H. Stroeher and P.A. Manning, 1988. Extracellular proteins of Vibrio cholerae: nucleotide sequence of the structural gene (hlyA) for the haemolysin of the haemolytic El Tor strain 017 and characterization of the hly A mutation in the non- haemolytic classical strain 569B. Mol. Microbiol, 2: 481-488.

23. Byun R., L.D. Elbourne, R. Lan and P.R. Reeves, 1999. Evolutionary relationships of pathogenic clones of Vibrio cholerae by sequence analysis of four housekeeping genes. Infect Immun, 67: 11161124.

24. Alm R.A. and P.A. Manning, 1990. Characterization of the $h l y \mathrm{~B}$ gene and its role in the production of the El Tor haemolysin of Vibrio cholerae O1. Mol. Microbiol, 4: 413-425.

25. Williams S.G. and P.A. Manning, 1991 Transcription of the Vibrio cholerae haemolysin gene, hlyA, and cloning of a positive regulatory locus, hlyU. Mol. Microbiol, 5: 2031-2038.

26. Williams S.G., S.R. Attridge and P.A. Manning, 1993. The transcriptional activator HlyU of Vibrio cholerae: nucleotide sequence and role in virulence gene expression. Mol Microbiol, 9: 751-760.

27. Rivera I.N., J. Chun, A. Huq, R.B. Sack and R.R. Colwell, 2001. Genotypes associated with virulence in environmental isolates of Vibrio cholerae. Appl Environ Microbiol, 67: 2421-2429.

28. Folgosa E., S. Mastrandrea, P. Cappuccinelli, S. Uzzau, P. Rappelli, M.J. Brian and M.M. Colombo, 2001. Molecular identification of pathogenicity genes and ERIC types in Vibrio cholerae $\mathrm{O} 1$ epidemic strains from Mozambique. Epidemiol Infect, 127: 17-25.

29. Janda J.M., C. Powers, R.G. Bryant and S.L. Abbott, 1988. Current perspectives on the epidemiology and pathogenesis of clinically significant Vibrio spp. Clin Microbiol Rev, 1: 245267.

30. Hertle R., M. Hilger, S. Weingardt-Kocher and I. Walev, 1999. Cytotoxic action of Serratia marcescens hemolysin on human epithelial cells. Infect Immun, 67: 817-825. 
31. Abrami L., M. Fivaz, P.E. Glauser, R.G. Parton and F.G. van der Goot, 1998. A pore-forming toxin interacts with a GPI-anchored protein and causes vacuolation of the endoplasmic reticulum. J Cell Biol, 140: 525-540.

32. Guyer D.M., S. Radulovic, F.E. Jones and H.L. Mobley, 2002. Sat, the secreted autotransporter toxin of uropathogenic Escherichia coli, is a vacuolating cytotoxin for bladder and kidney epithelial cells. Infect Immun, 70: 4539-4546.

33. Nishibuchi M. and J.B. Kaper, 1995. Thermostable direct hemolysin gene of Vibrio parahaemolyticus: a virulence gene acquired by a marine bacterium. Infect Immun, 63: 2093-2099.
34. Clark J.M., 1988. Novel non-templated nucleotide addition reactions catalyzed by prokaryotic and eukaryotic DNA polymerases. Nucleic Acids Res, 16: 9677-9686.

35. Gutiérrez M.G., H-A. Saka, I. Chinen, F.C. Zoppino, T. Yoshimori, J.L. Bocco and M.I. Colombo, 2007. Protective role of autophagy against Vibrio cholerae cytolysin a pore forming toxin of V. cholerae. Proc Nat Acad Sci USA, 104: 1829-1834. 